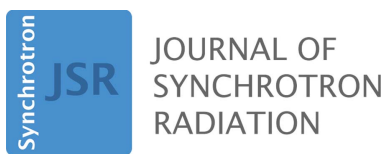

ISSN 1600-5775

Keywords: X-ray free-electron lasers; XFELs

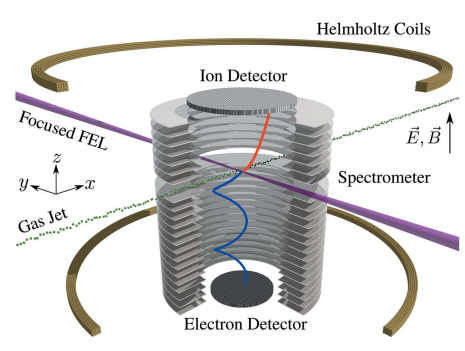

(C) 2020 International Union of Crystallography

\section{Foreword to the special virtual issue on $X$-ray free-electron lasers}

\author{
Paul H. Fuoss, ${ }^{a}$ Ilme Schlichting, ${ }^{\text {b* }}{ }^{\text {Thomas }}$ Tschentscher $^{\mathrm{c}}$ and Makina Yabashid

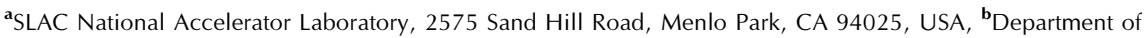 \\ Biomolecular Mechanisms, Max Planck Institute for Medical Research, Jahnstrasse 29, 69120 Heidelberg, Germany, \\ ' European XFEL, Notkestrasse 85, 22607 Hamburg, Germany, and ${ }^{\mathbf{d}}$ RIKEN SPring-8 Center, Kouto 1-1-1, Sayo, \\ Hyogo 679-5148, Japan. *Correspondence e-mail: jsr@mpimf-heidelberg.mpg.de
}

The last five years have seen a remarkable explosion in both the availability and capabilities of X-ray free-electron lasers (XFELs). When the first XFEL turned on at DESY's Tesla Test Facility twenty years ago, a host of challenges and uncertainties faced the X-ray community. Many of these challenges have been overcome allowing the field to blossom and grow. Following the demonstration of hard X-ray lasing at LCLS a decade ago, the number of facilities rapidly expanded and routine user operations were available at FERMI, FLASH, LCLS and SACLA in 2015. Since then three new XFELs, PALXFEL, European XFEL and SwissFEL, have started operations and the FLASH facility was significantly expanded. At the same time new sources were being commissioned, the performance of XFELs was being expanded with new operating modes (e.g. multi-bunch and two-color), better X-ray optics, enhanced control systems, and detectors purpose built for XFEL operations. There is little evidence that this rapid growth in capabilities is slowing; there are new instruments being built at all of the XFELs, and new scientific techniques and applications being developed at a startling rate. In addition, following the first high repetition rate accelerators at FLASH and European XFEL this technology now becomes much more widespread with the imminent commissioning of the LCLS-II, the planned LCLS-II-HE upgrade at SLAC and the SHINE project in Shanghai.

Considering this rapid progress, it is timely for another special FEL-related issue in Journal of Synchrotron Radiation, following the first one published in May 2015. The current virtual edition (https://journals.iucr.org/special_issues/2020/xfels/index.html) provides information to guide both X-ray experts and new users. The edition features articles describing a variety of sources and facilities, including FLASH, LCLS, SACLA, SwissFEL, PAL and EuXFEL, that give insight into the current status, development plans and scientific directions at these facilities. Papers focused on individual instruments provide detailed information into new experimental capabilities along with recent results highlighting the scientific opportunities being developed at XFELs. Overviews of the design and performance of important subsystems, for example laser systems for timeresolved measurements, give critical information to plan successful XFEL experiments. In particular, a number of papers focus on the infrastructure requirements and challenges at the European XFEL, the first of the next generation of megahertz repetition rate XFEL producing hard ( $\lesssim 1 \AA$ wavelength) X-rays. Finally, new X-ray scientific methods are constantly being ported to, and developed at, FEL sources. This edition highlights a few of these capabilities including spectroscopy techniques.

In this rapidly evolving experimental environment, this virtual edition is a snapshot of the field circa 2018. Unfortunately, it cannot be comprehensive because the field is large, diverse and complex, but, more importantly, because the field is rapidly evolving. Nevertheless, it provides information to the XFEL community today and context for the future. We hope you find it valuable. 